\title{
Applications of differential subordinations involving a generalized fractional differintegral operator
}

Hanaa M. Zayed ${ }^{1,2^{*}}$ and Teodor Bulboacă ${ }^{3}$

"Correspondence:

hanaa_zayed42@yahoo.com

'Department of Mathematics, ITAM,

Mexico City, Mexico

${ }^{2}$ Department of Mathematics,

Faculty of Science, Menofia

University, Shebin Elkom, Egypt

Full list of author information is

available at the end of the article

\begin{abstract}
Using the third-order differential subordination basic results, we introduce certain classes of admissible functions and investigate some applications of third-order differential subordination for $p$-valent functions associated with generalized fractional differintegral operator.
\end{abstract}

MSC: $30 C 45 ; 30 C 50$

Keywords: Analytic function; $p$-valent functions; Third-order differential subordination; Admissible functions; Generalized fractional derivative operator; Generalized fractional integral operator

\section{Introduction and preliminaries}

Let $\mathcal{H}(\mathbb{U})$ denote the space of analytic functions in the unit disk $\mathbb{U}:=\{z \in \mathbb{C}:|z|<1\}$, $\mathcal{H}[a, n]$ denote the subclass of functions $f \in \mathcal{H}(\mathbb{U})$ of the form

$$
f(z)=a+a_{n} z^{n}+a_{n+1} z^{n+1}+\cdots, \quad z \in \mathbb{U}, \quad(a \in \mathbb{C}, n \in \mathbb{N}:=\{1,2, \ldots\}),
$$

and $\mathcal{H}_{p}:=\mathcal{H}[0, p]$. Also, let $\mathcal{A}(p)$ be the subclass of functions $f \in \mathcal{H}(\mathbb{U})$ of the form

$$
f(z)=z^{p}+\sum_{n=1}^{\infty} a_{p+n} z^{p+n}, \quad z \in \mathbb{U}, \quad(p \in \mathbb{N})
$$

and set $\mathcal{A}:=\mathcal{A}(1)$.

For two functions $f, g \in \mathcal{H}(\mathbb{U})$, we say that the function $f$ is subordinate to $g$, written $f \prec g$, if there exists a Schwarz function $w$, analytic in $\mathbb{U}$ with $w(0)=0$ and $|w(z)|<1$ such that $f(z)=g(w(z))$ for all $z \in \mathbb{U}$. Furthermore, if the function $g$ is univalent in $\mathbb{U}$, then we have the following equivalence (see [9] and [12]):

$$
f(z) \prec g(z) \quad \Leftrightarrow \quad f(0)=g(0) \text { and } f(\mathbb{U}) \subset g(\mathbb{U})
$$

(c) The Author(s) 2019. This article is distributed under the terms of the Creative Commons Attribution 4.0 International License (http://creativecommons.org/licenses/by/4.0/), which permits unrestricted use, distribution, and reproduction in any medium, provided you give appropriate credit to the original author(s) and the source, provide a link to the Creative Commons license, and indicate if changes were made. 
Let $\phi(r, s, t ; z): \mathbb{C}^{4} \times \mathbb{U} \rightarrow \mathbb{C}$ and $h$ be univalent in $\mathbb{U}$. If $p$ is analytic in $\mathbb{U}$ and satisfies the third-order differential subordination

$$
\phi\left(p(z), z p^{\prime}(z), z^{2} p^{\prime \prime}(z), z^{3} p^{\prime \prime \prime}(z) ; z\right) \prec h(z)
$$

then $p$ is called a solution of the differential subordination (1.2). The univalent function $q$ is said to be a dominant of (1.2) if $p(z) \prec q(z)$ for all $p$ that satisfy (1.2). A dominant $\widetilde{q}$ is called the best dominant if $\widetilde{q}(z) \prec q(z)$ for all dominants $q$ of (1.2).

We recall here the following generalized fractional integral and generalized fractional derivative operators due to Srivastava et al. [22] (see also [16, 17]).

Definition 1.1 ([22, Definition 3]) For $\lambda>0$ and $\mu, \eta$ real numbers, the Srivastava-SaigoOwa hypergeometric fractional integral operator $\mathrm{I}_{0, z}^{\lambda, \mu, \eta}$ is defined by

$$
I_{0, z}^{\lambda, \mu, \eta} f(z)=\frac{z^{-\lambda-\mu}}{\Gamma(\lambda)} \int_{0}^{z}(z-\zeta)^{\lambda-1} F\left(\mu+\lambda,-\eta ; \lambda ; 1-\frac{\zeta}{z}\right) f(\zeta) d \zeta
$$

where $f$ is an analytic function in a simply-connected region of the complex $z$-plane containing the origin with the order $f(z)=O\left(|z|^{\varepsilon}\right), z \rightarrow 0$, where $\varepsilon>\max \{0, \mu-\eta\}-1$ and the multiplicity of $(z-\zeta)^{\lambda-1}$ is removed by requiring $\log (z-\zeta)$ to be real when $z-\zeta>0$. Also, $\Gamma$ is the well-known gamma function, while the function $F$ denotes the Gauss hypergeometric function, that is,

$$
{ }_{2} F_{1}(a, b ; c: z)=\sum_{n=0}^{\infty} \frac{(a)_{n}(b)_{n}}{(c)_{n} n !} z^{n}, \quad|z|<1
$$

and its analytic continuation into $|\arg (1-z)|<\pi$ and $(a)_{n}=\Gamma(a+n) / \Gamma(a)$.

Definition 1.2 Under the hypotheses of Definition 1.1, the Srivastava-Saigo-Owa hypergeometric fractional derivative operator $J_{0, z}^{\lambda, \mu, \eta}$ is defined by

$$
J_{0, z}^{\lambda, \mu, \eta} f(z)= \begin{cases}\frac{d}{d z}\left[\frac{z^{\lambda-\mu} \int_{0}^{z}(z-\zeta)^{-\lambda} f(\zeta)_{2} F_{1}\left(\mu-\lambda, 1-\eta ; 1-\lambda ; 1-\frac{\zeta}{z}\right) d \zeta}{\Gamma(1-\lambda)}\right] & \text { if } 0 \leq \lambda<1, \\ \frac{d^{n}}{d z^{n}} J_{0, z}^{\lambda-n, \mu, \eta} f(z), & \text { if } n \leq \lambda<n+1, n \in \mathbb{N},\end{cases}
$$

where the multiplicity of $(z-\zeta)^{-\lambda}$ is removed as in Definition 1.1.

We note that

$$
I_{0, z}^{\lambda,-\lambda, \eta} f(z)=D_{z}^{-\lambda} f(z), \quad \text { if } \lambda>0, \quad \text { and } \quad J_{0, z}^{\lambda, \lambda, \eta} f(z)=D_{z}^{\lambda} f(z), \quad \text { if } 0 \leq \lambda<1
$$

where $D_{z}^{-\lambda}$ denotes the fractional integral operator, and $D_{z}^{\lambda}$ denotes the fractional derivative operator studied by Owa [13]. 
In relation to the Srivastava-Saigo-Owa hypergeometric fractional derivative operator, Goyal and Prajapat [10] (see also [15]) defined the operator

$$
S_{0, z}^{\lambda, \mu, \eta, p} f(z)=\left\{\begin{array}{c}
\frac{\Gamma(p+1-\mu) \Gamma(p+1-\lambda+\eta)}{\Gamma(p+1) \Gamma(p+1-\mu+\eta)} z^{\mu} J_{0, z}^{\lambda, \mu, \eta} f(z) \\
\text { if } 0 \leq \lambda<\eta+p+1, z \in \mathbb{U} \\
\frac{\Gamma(p+1-\mu) \Gamma(p+1-\lambda+\eta)}{\Gamma(p+1) \Gamma(p+1-\mu+\eta)} z^{\mu} I_{0, z}^{-\lambda, \mu, \eta} f(z) \\
\text { if }-\infty<\lambda<0, z \in \mathbb{U} .
\end{array}\right.
$$

Thus, for a function $f \in \mathcal{A}(p)$ of the form (1.1), we have

$$
\begin{aligned}
S_{0, z}^{\lambda, \mu, \eta, p} f(z) & =z^{p}{ }_{3} F_{2}(1,1+p, 1+p+\eta-\mu ; 1+p-\mu, 1+p+\eta-\lambda ; z) * f(z) \\
& =z^{p}+\sum_{n=1}^{\infty} \frac{(p+1)_{n}(p+1-\mu+\eta)_{n}}{(p+1-\mu)_{n}(p+1-\lambda+\eta)_{n}} a_{p+n} z^{p+n}, \quad z \in \mathbb{U} \\
(p \in \mathbb{N}, \mu, \eta \in \mathbb{R}, \mu<p+1, \infty<\lambda<\eta+p+1) &
\end{aligned}
$$

where ${ }_{q} F_{s}$, with $q \leq s+1$ and $q, s \in \mathbb{N}_{0}:=\mathbb{N} \cup\{0\}$, is the well-known generalized hypergeometric function (for more details, see [14] and [20]) and $(v)_{n}$ is the Pochhammer symbol defined by

$$
(v)_{n}= \begin{cases}1, & \text { if } n=0 \\ v(v+1)(v+2) \cdots(v+n-1), & \text { if } n \in \mathbb{N} .\end{cases}
$$

Let

$$
\begin{aligned}
& G_{p, \eta, \mu}^{\lambda}(z)=z^{p}+\sum_{n=1}^{\infty} \frac{(p+1)_{n}(p+1-\mu+\eta)_{n}}{(p+1-\mu)_{n}(p+1-\lambda+\eta)_{n}} z^{p+n}, \quad z \in \mathbb{U}, \\
& (p \in \mathbb{N}, \mu, \eta \in \mathbb{R}, \mu<p+1,-\infty<\lambda<\eta+p+1)
\end{aligned}
$$

and define the new function $\left[G_{p, \eta, \mu}^{\lambda}\right]^{-1}$ by means of the Hadamard (or convolution) product

$$
G_{p, \eta, \mu}^{\lambda}(z) *\left[G_{p, \eta, \mu}^{\lambda}(z)\right]^{-1}=\frac{z^{p}}{(1-z)^{\delta+p}}, \quad z \in \mathbb{U}, \quad(\delta>-p) .
$$

Using the above defined function, Tang et al. [24] (see also Aouf et al. [4, 7] and [8]) defined the operator $H_{p, \eta, \mu}^{\lambda, \delta}: \mathcal{A}(p) \rightarrow \mathcal{A}(p)$ by

$$
H_{p, \eta, \mu}^{\lambda, \delta} f(z)=\left[G_{p, \eta, \mu}^{\lambda}(z)\right]^{-1} * f(z)
$$

It is easy to check that, for a function $f \in \mathcal{A}(p)$ of the form (1.1), we have

$$
H_{p, \eta, \mu}^{\lambda, \delta} f(z)=z^{p}+\sum_{n=1}^{\infty} \frac{(\delta+p)_{n}(p+1-\mu)_{n}(p+1-\lambda+\eta)_{n}}{(1)_{n}(p+1)_{n}(p+1-\mu+\eta)_{n}} a_{p+n} z^{p+n}, \quad z \in \mathbb{U} .
$$


For $k \in \mathbb{N}_{0}$ and $\zeta>0$, Aouf et al. [6] defined the operator $\mathcal{N}_{p, \lambda, \mu, \eta}^{m, \delta, \zeta}: \mathcal{A}(p) \rightarrow \mathcal{A}(p)$ as follows:

$$
\begin{aligned}
\mathcal{N}_{p, \lambda, \mu, \eta}^{0, \delta, \zeta} f(z) & =H_{p, \eta, \mu}^{\lambda, \delta} f(z), \\
\mathcal{N}_{p, \lambda, \mu, \eta}^{1, \delta, \zeta} f(z) & =: \mathcal{N}_{p, \lambda, \mu, \eta}^{\delta, \zeta} f(z)=(1-\zeta) H_{p, \eta, \mu}^{\lambda, \delta} f(z)+\zeta \frac{z}{p}\left[H_{p, \eta, \mu}^{\lambda, \delta} f(z)\right]^{\prime} \\
& =z^{p}+\sum_{n=1}^{\infty} \frac{p+\zeta n}{p} \frac{(\delta+p)_{n}(p+1-\mu)_{n}(p+1-\lambda+\eta)_{n}}{(1)_{n}(p+1)_{n}(p+1-\mu+\eta)_{n}} a_{p+n} z^{p+n},
\end{aligned}
$$

and, in general,

$$
\begin{aligned}
\mathcal{N}_{p, \lambda, \mu, \eta}^{k, \delta, \zeta} f(z) & =: \mathcal{N}_{p, \lambda, \mu, \eta}^{\delta, \zeta}\left(\mathcal{N}_{p, \lambda, \mu, \eta}^{k-1, \delta, \zeta} f(z)\right) \\
& =z^{p}+\sum_{n=1}^{\infty}\left(\frac{p+\zeta n}{p}\right)^{k} \frac{(\delta+p)_{n}(p+1-\mu)_{n}(p+1-\lambda+\eta)_{n}}{(1)_{n}(p+1)_{n}(p+1-\mu+\eta)_{n}} a_{p+n} z^{p+n}
\end{aligned}
$$

Remark 1.1 1. We note that the operator $\mathcal{N}_{p, \lambda, \mu, \eta}^{m, \delta, \zeta}$ generalizes many other remarkable previously studied operators, like:

(i) $\quad \mathcal{N}_{p, \lambda, \mu, \eta, \eta}^{0, \delta, \zeta} f(z)=: H_{p, \eta, \mu}^{\lambda, \delta} f(z) \quad($ see [24]);

(ii) $\mathcal{N}_{p, p, p, 0}^{k, 1, \zeta} f(z)=: D_{\zeta, p}^{k} f(z) \quad$ (see [3]);

(iii) $\mathcal{N}_{p, p, p, 0}^{k, 1,1} f(z)=: D_{p}^{k} f(z) \quad$ (see [5] and [11]);

(iv) $\mathcal{N}_{1,1,1,0}^{k, 1, \zeta} f(z)=: D_{\zeta}^{k} f(z) \quad($ see $[1])$;

(v) $\mathcal{N}_{1,1,1,0}^{k, 1,1} f(z)=: D^{k} f(z) \quad($ see $[18])$.

2. Also, we remark the following special cases of this operator:

(i) $\mathcal{N}_{p, \lambda, \mu, \eta}^{k, 1, \zeta} f(z)=: \mathcal{N}_{p, \lambda, \mu, \eta}^{k, \zeta} f(z)$

$$
=z^{p}+\sum_{n=1}^{\infty}\left(\frac{p+\zeta n}{p}\right)^{k} \frac{(p+1-\mu)_{n}(p+1-\lambda+\eta)_{n}}{(1)_{n}(p+1-\mu+\eta)_{n}} a_{p+n} z^{p+n}
$$

(ii) $\mathcal{N}_{p, \lambda, \lambda, n}^{k, \delta, \zeta} f(z)=: \mathcal{N}_{p, \lambda}^{k, \delta, \zeta} f(z)=z^{p}+\sum_{n=1}^{\infty}\left(\frac{p+\zeta n}{p}\right)^{k} \frac{(\delta+p)_{n}(p+1-\lambda)_{n}}{(1)_{n}(p+1)_{n}} a_{p+n} z^{p+n}$;

(iii) $\mathcal{N}_{p, \mu, \mu, \eta}^{k, 1, \zeta} f(z)=: \mathcal{N}_{p, \mu}^{k, \delta, \zeta} f(z)=z^{p}+\sum_{n=1}^{\infty}\left(\frac{p+\zeta n}{p}\right)^{k} \frac{(\delta+p)_{n}(p+1-\mu)_{n}}{(1)_{n}(p+1)_{n}} a_{p+n} z^{p+n}$.

Moreover, it is easy to verify from (1.3) that

$$
\begin{aligned}
& \zeta z\left(\mathcal{N}_{p, \lambda, \mu, \eta}^{k, \delta, \zeta} f(z)\right)^{\prime}=p \mathcal{N}_{p, \lambda, \mu, \eta}^{k+1, \delta, \zeta} f(z)-p(1-\zeta) \mathcal{N}_{p, \lambda, \mu, \eta}^{k, \delta, \zeta} f(z) \quad \text { for } \zeta>0, \\
& z\left(\mathcal{N}_{p, \lambda+1, \mu, \eta}^{k, \delta, \zeta} f(z)\right)^{\prime}=(p+\eta-\lambda) \mathcal{N}_{p, \lambda, \mu, \eta}^{k, \delta, \zeta} f(z)-(\eta-\lambda) \mathcal{N}_{p, \lambda+1, \mu, \eta}^{k, \delta, \zeta} f(z),
\end{aligned}
$$

and

$$
z\left(\mathcal{N}_{p, \lambda, \mu, \eta}^{k, \delta, \zeta} f(z)\right)^{\prime}=(\delta+p) \mathcal{N}_{p, \lambda, \mu, \eta}^{k, \delta+1, \zeta} f(z)-\delta \mathcal{N}_{p, \lambda, \mu, \eta}^{k, \delta, \zeta} f(z)
$$


To obtain our results, we need to use the following definitions and theorems.

Definition 1.3 ([2, p. 441]) Let $\mathcal{Q}$ be the set of all functions $q$ that are analytic and univalent on $\overline{\mathbb{U}} \backslash E(q)$, where

$$
E(q)=\left\{\xi \in \partial \mathbb{U}: \lim _{z \rightarrow \xi} q(z)=\infty\right\}
$$

and are such that

$$
\min \left|q^{\prime}(\xi)\right|=\rho>0 \quad \text { for } \xi \in \partial \mathbb{U} \backslash E(q) .
$$

Further, let the subclass of $\mathcal{Q}$ for which $q(0)=a$ be denoted by $\mathcal{Q}(a)$ and $\mathcal{Q}(0)=: \mathcal{Q}_{0}$.

Definition 1.4 ([2, p. 449]) Let $\Omega$ be a subset of $\mathbb{C}, q \in \mathcal{Q}$ and $n \geq 2$. The class of admissible operators $\Psi_{n}[\Omega, q]$ consists of those functions $\psi: \mathbb{C}^{4} \times \mathbb{U} \rightarrow \mathbb{C}$ that satisfy the following admissibility condition

$$
\psi(r, s, t, u ; z) \notin \Omega,
$$

whenever

$$
r=q(w), \quad s=n w q^{\prime}(w), \quad \operatorname{Re} \frac{t}{s}+1 \geq n \operatorname{Re}\left(\frac{w q^{\prime \prime}(w)}{q^{\prime}(w)}+1\right),
$$

and

$$
\operatorname{Re} \frac{u}{s} \geq n^{2} \operatorname{Re} \frac{w^{2} q^{\prime \prime \prime}(w)}{q^{\prime}(w)}, \quad z \in \mathbb{U}, w \in \partial \mathbb{U} \backslash E(q) .
$$

Lemma 1.1 ([2, p. 449]) Let $\Omega$ be a subset of $\mathbb{C}, \psi \in \Psi_{n}[\Omega, q]$ and $p \in \mathcal{H}[a, n]$ with $n \geq 2$. If $q \in \mathcal{Q}(a)$ and satisfies the following conditions

$$
\operatorname{Re} \frac{w q^{\prime \prime}(w)}{q^{\prime}(w)} \geq 0 \quad \text { and } \quad\left|\frac{z p^{\prime}(z)}{q^{\prime}(w)}\right| \leq n, \quad z \in \mathbb{U}, w \in \partial \mathbb{U} \backslash E(q),
$$

then

$$
\psi\left(p(z), z p^{\prime}(z), z^{2} p^{\prime \prime}(z), z^{3} p^{\prime \prime \prime}(z) ; z\right) \in \Omega, \quad z \in \mathbb{U},
$$

implies $p(z) \prec q(z)$.

The aim of the present article is to use the recent works by Tang et al. (see [25, 26]) to systematically investigate the third-order differential subordination general theory to a suitable classes of admissible functions. We obtained new results for a wide class of operators defined by convolution products with Srivastava-Saigo-Owa generalized fractional integral and generalized fractional derivative operators. Our results give interesting new properties and, together with other papers that appeared in the last years, could emphasize the perspective of the importance of the third-order subordination theory and the Srivastava-Saigo-Owa generalized operators. 
We emphasize that in recent years, several authors obtained many interesting results involving different linear and convolution operators associated with second-order differential subordinations (see $[19,23])$ and regarding the third-order differential subordinations (see [21]) for the above mentioned operator.

\section{Main results}

Unless otherwise mentioned, we assume throughout this paper that $f \in \mathcal{A}(p), \zeta>0, p \geq 2$, $w \in \partial \mathbb{U} \backslash E(q), \theta \in[0,2 \pi]$ and $z \in \mathbb{U}$.

Definition 2.1 Let $\Omega$ be a subset of $\mathbb{C}$ and $q \in \mathcal{Q}_{0}$. The class of admissible operators $\Phi_{p}[\Omega, q]$ consists of those functions $\phi: \mathbb{C}^{4} \times \mathbb{U} \rightarrow \mathbb{C}$ that satisfy the following admissibility condition:

$$
\phi(\alpha, \beta, \gamma, \varepsilon ; z) \notin \Omega
$$

whenever

$$
\begin{aligned}
& \alpha=q(w), \quad \beta=w \zeta q^{\prime}(w)+(1-\zeta) q(w), \\
& \operatorname{Re}\left(\frac{p}{\zeta} \frac{\gamma+(1-\zeta)\left(1-\zeta+\frac{\zeta}{p}\right) \alpha-\left(2-2 \zeta+\frac{\zeta}{p}\right) \beta}{\beta-(1-\zeta) \alpha}+1\right) \geq p \operatorname{Re}\left(\frac{w q^{\prime \prime}(w)}{q^{\prime}(w)}+1\right),
\end{aligned}
$$

and

$$
\left.\begin{array}{l}
\frac{p^{2}}{\zeta^{2}} \operatorname{Re}\left(\begin{array}{c}
\varepsilon-3\left(1-\zeta+\frac{\zeta}{p}\right) \gamma-(1-\zeta)\left(1-\zeta+\frac{\zeta}{p}\right)\left(1-\zeta+\frac{2 \zeta}{p}\right) \alpha \\
+\left[2\left(1-\zeta+\frac{\zeta}{p}\right)\left(2-2 \zeta+\frac{\zeta}{p}\right)-(1-\zeta)^{2}\right] \beta
\end{array}\right) \\
\beta-(1-\zeta) \alpha
\end{array}\right)
$$

Theorem 2.1 Let $\Omega$ be a subset of $\mathbb{C}$ and $\phi \in \Phi_{p}[\Omega, q]$. If $q \in \mathcal{Q}_{0}$ satisfies the following conditions:

$$
\operatorname{Re} \frac{w q^{\prime \prime}(w)}{q^{\prime}(w)} \geq 0 \quad \text { and } \quad\left|\frac{z\left(\mathcal{N}_{p, \lambda, \mu, \eta}^{k, \delta, \zeta} f(z)\right)^{\prime}}{q^{\prime}(w)}\right| \leq p
$$

then

$$
\left\{\phi\left(\mathcal{N}_{p, \lambda, \mu, \eta, \eta}^{k, \delta, \zeta} f(z), \mathcal{N}_{p, \lambda, \mu, \eta}^{k+1, \delta, \zeta} f(z), \mathcal{N}_{p, \lambda, \mu, \eta}^{k+2, \delta, \zeta} f(z), \mathcal{N}_{p, \lambda, \mu, \eta}^{k+3, \delta, \zeta} f(z) ; z\right): z \in \mathbb{U}\right\} \subset \Omega
$$

implies

$$
\mathcal{N}_{p, \lambda, \mu, \eta}^{k, \delta, \zeta} f(z) \prec q(z) .
$$

Proof Defining the function $p$ by

$$
p(z)=\mathcal{N}_{p, \lambda, \mu, \eta}^{k, \delta, \zeta} f(z)
$$


then $p$ is analytic in $\mathbb{U}$. Differentiating three times (2.3) with respect to $z$ and using (1.4), we obtain the following relations, respectively:

$$
\begin{aligned}
\mathcal{N}_{p, \lambda, \mu, \eta}^{k+1, \delta, \zeta} f(z)= & \frac{\zeta}{p} z p^{\prime}(z)+(1-\zeta) p(z) \\
\mathcal{N}_{p, \lambda, \mu, \eta}^{k+2, \delta, \zeta} f(z)= & \frac{\zeta^{2}}{p^{2}} z^{2} p^{\prime \prime}(z)+\frac{\zeta}{p}\left(2-2 \zeta+\frac{\zeta}{p}\right) z p^{\prime}(z)+(1-\zeta)^{2} p(z) \\
\mathcal{N}_{p, \lambda, \mu, \eta}^{k+3, \delta, \zeta} f(z)= & \frac{\zeta^{3}}{p^{3}} z^{3} p^{\prime \prime \prime}(z)+\frac{3 \zeta^{2}}{p^{2}}\left(1-\zeta+\frac{\zeta}{p}\right) z^{2} p^{\prime \prime}(z) \\
& +\frac{\zeta}{p}\left[(1-\zeta)^{2}+\left(1-\zeta+\frac{\zeta}{p}\right)\left(2-2 \zeta+\frac{\zeta}{p}\right)\right] z p^{\prime}(z)+(1-\zeta)^{3} p(z)
\end{aligned}
$$

Letting

$$
\begin{aligned}
\alpha(r, s, t, u)= & r, \quad \beta(r, s, t, u)=\frac{\zeta}{p} s+(1-\zeta) r \\
\gamma(r, s, t, u)= & \frac{\zeta^{2}}{p^{2}} t+\frac{\zeta}{p}\left(2-2 \zeta+\frac{\zeta}{p}\right) s+(1-\zeta)^{2} r \\
\varepsilon(r, s, t, u)= & \frac{\zeta^{3}}{p^{3}} u+\frac{3 \zeta^{2}}{p^{2}}\left(1-\zeta+\frac{\zeta}{p}\right) t \\
& +\frac{\zeta}{p}\left[(1-\zeta)^{2}+\left(1-\zeta+\frac{\zeta}{p}\right)\left(2-2 \zeta+\frac{\zeta}{p}\right)\right] s+(1-\zeta)^{3} r
\end{aligned}
$$

we will define the transformation $\psi: \mathbb{C}^{4} \times \mathbb{U} \rightarrow \mathbb{C}$ by

$$
\psi(r, s, t, u ; z)=\phi(\alpha, \beta, \gamma, \varepsilon ; z)
$$

Then, using relations (2.3), (2.4), (2.5) and (2.6), we have

$$
\begin{aligned}
\psi & \left(p(z), z p^{\prime}(z), z^{2} p^{\prime \prime}(z), z^{3} p^{\prime \prime \prime}(z) ; z\right) \\
\quad & \phi \phi\left(\mathcal{N}_{p, \lambda, \mu, \eta}^{k, \delta, \zeta} f(z), \mathcal{N}_{p, \lambda, \mu, \eta}^{k+1, \delta, \zeta} f(z), \mathcal{N}_{p, \lambda, \mu, \eta}^{k+2, \delta, \zeta} f(z), \mathcal{N}_{p, \lambda, \mu, \eta}^{k+3, \delta, \zeta} f(z) ; z\right)
\end{aligned}
$$

Since

$$
\frac{t}{s}+1=\frac{p}{\zeta} \frac{\gamma+(1-\zeta)\left(1-\zeta+\frac{\zeta}{p}\right) \alpha-\left(2-2 \zeta+\frac{\zeta}{p}\right) \beta}{\beta-(1-\zeta) \alpha}+1,
$$

and

$$
\frac{u}{s}=\frac{p^{2}}{\zeta^{2}}\left(\begin{array}{c}
\varepsilon-3\left(1-\zeta+\frac{\zeta}{p}\right) \gamma-(1-\zeta)\left(1-\zeta+\frac{\zeta}{p}\right)\left(1-\zeta+\frac{2 \zeta}{p}\right) \alpha \\
+\left[2\left(1-\zeta+\frac{\zeta}{p}\right)\left(2-2 \zeta+\frac{\zeta}{p}\right)-(1-\zeta)^{2}\right] \beta \\
\beta-(1-\zeta) \alpha
\end{array}\right)
$$

the admissibility condition for $\phi \in \Phi_{p}[\Omega, q]$ of Definition 2.1 is equivalent to the admissibility condition for $\psi$ as given in Definition 1.4. Thus, the proof follows from Lemma 1.1 by setting $a=0, n=p$ and $a_{p}=1$. 
The next result is an extension of Theorem 2.1 for the case when the behavior of $q$ on $\partial \mathbb{U}$ is unknown.

Corollary 2.1 Let $\Omega$ be a subset of $\mathbb{C}$ and $q$ be univalent in $\mathbb{U}$ with $q \in \mathcal{Q}_{0}$. Let $\phi \in$ $\Phi_{p}\left[\Omega, q_{\rho}\right]$ for some $\rho \in(0,1)$, where $q_{\rho}(z)=q(\rho z)$. If $q_{\rho}$ satisfies the following conditions:

$$
\operatorname{Re} \frac{w q_{\rho}^{\prime \prime}(w)}{q_{\rho}^{\prime}(w)} \geq 0 \quad \text { and } \quad\left|\frac{z\left(\mathcal{N}_{p, \lambda, \mu, \eta}^{k, \delta, \zeta} f(z)\right)^{\prime}}{q_{\rho}^{\prime}(w)}\right| \leq p, \quad w \in \partial \mathbb{U} \backslash E\left(q_{\rho}\right)
$$

then

$$
\left\{\phi\left(\mathcal{N}_{p, \lambda, \mu, \eta}^{k, \delta, \zeta} f(z), \mathcal{N}_{p, \lambda, \mu, \eta}^{k+1, \delta, \zeta} f(z), \mathcal{N}_{p, \lambda, \mu, \eta}^{k+2, \delta, \zeta} f(z), \mathcal{N}_{p, \lambda, \mu, \eta}^{k+3, \delta, \zeta} f(z) ; z\right): z \in \mathbb{U}\right\} \subset \Omega
$$

implies

$$
\mathcal{N}_{p, \lambda, \mu, \eta, \eta}^{k, \delta, \zeta} f(z) \prec q(z)
$$

Proof From Theorem 2.1 we obtain $\mathcal{N}_{p, \lambda, \mu, \eta}^{k, \delta, \zeta} f(z) \prec q_{\rho}(z)$ and since $q_{\rho}(z) \prec q(z)$, we conclude that $\mathcal{N}_{p, \lambda, \mu, \eta}^{k, \delta, \zeta} f(z) \prec q(z)$.

If $\Omega \neq \mathbb{C}$ is a simply connected domain, then $\Omega=h(\mathbb{U})$ for some conformal mapping $h$ of $\mathbb{U}$ onto $\Omega$. In this case, the class $\Phi_{p}[h(\mathbb{U}), q]$ will be written as $\Phi_{p}[h, q]$. The following two results are direct consequences of Theorem 2.1 and Corollary 2.1.

Corollary 2.2 Let $\phi \in \Phi_{p}[h, q]$, where $h$ is univalent in $\mathbb{U}$ and suppose that $q \in \mathcal{Q}_{0}$ satisfies conditions (2.1). Then

$$
\phi\left(\mathcal{N}_{p, \lambda, \mu, \mu, \eta}^{k, \delta, \zeta} f(z), \mathcal{N}_{p, \lambda, \mu, \eta}^{k+1, \delta, \zeta} f(z), \mathcal{N}_{p, \lambda, \mu, \eta}^{k+2, \delta, \zeta} f(z), \mathcal{N}_{p, \lambda, \mu, \eta}^{k+3, \delta, \zeta} f(z) ; z\right) \prec h(z)
$$

implies

$$
\mathcal{N}_{p, \lambda, \mu, \eta}^{k, \delta, \zeta} f(z) \prec q(z)
$$

Corollary 2.3 Let $q$ be univalent in $\mathbb{U}$ with $q \in \mathcal{Q}_{0}$ and $\phi \in \Phi_{p}\left[h, q_{\rho}\right]$ for some $\rho \in(0,1)$, where $q_{\rho}(z)=q(\rho z)$. If $q_{\rho}$ satisfies conditions (2.9), then the subordination (2.10) implies that

$$
\mathcal{N}_{p, \lambda, \mu, \eta}^{k, \delta, \zeta} f(z) \prec q(z)
$$

We next show the relation between the best dominant of a differential subordination and the solution of a corresponding differential equation.

Corollary 2.4 Let $h$ be univalent in $\mathbb{U}$ and $\psi$ be given by (2.8) where $\phi \in \Phi_{p}[h, q]$. Suppose that the differential equation

$$
\psi\left(q(z), z q^{\prime}(z), z^{2} q^{\prime \prime}(z), z^{3} q^{\prime \prime \prime}(z) ; z\right)=h(z)
$$


has a solution $q$ with $q \in \mathcal{Q}_{0}$ that satisfies conditions (2.1). Then subordination (2.10) implies that

$$
\mathcal{N}_{p, \lambda, \mu, \eta}^{k, \delta, \zeta} f(z) \prec q(z)
$$

and $q$ is the best dominant of (2.10).

Proof Since

$$
\begin{aligned}
\phi & \left(\mathcal{N}_{p, \lambda, \mu, \eta}^{k, \delta, \zeta} f(z), \mathcal{N}_{p, \lambda, \mu, \eta}^{k+1, \delta, \zeta} f(z), \mathcal{N}_{p, \lambda, \mu, \eta}^{k+2, \delta, \zeta} f(z), \mathcal{N}_{p, \lambda, \mu, \eta}^{k+3, \delta, \zeta} f(z) ; z\right) \\
& =\psi\left(p(z), z p^{\prime}(z), z^{2} p^{\prime \prime}(z), z^{3} p^{\prime \prime \prime}(z) ; z\right) \prec h(z),
\end{aligned}
$$

then $p$ is a solution of (2.11), and from Corollary 2.2 we obtain that $p(z) \prec q(z)$, that is, $q$ is a dominant of (2.11). Also,

$$
\begin{aligned}
\phi & \left(\mathcal{N}_{p, \lambda, \mu, \eta}^{k, \delta, \zeta} f(z), \mathcal{N}_{p, \lambda, \mu, \eta}^{k+1, \delta, \zeta} f(z), \mathcal{N}_{p, \lambda, \mu, \eta}^{k+2, \delta, \zeta} f(z), \mathcal{N}_{p, \lambda, \mu, \eta}^{k+3, \delta, \zeta} f(z) ; z\right) \\
\quad & =\psi\left(p(z), z p^{\prime}(z), z^{2} p^{\prime \prime}(z), z^{3} p^{\prime \prime \prime}(z) ; z\right) \prec h(z)=\psi\left(q(z), z q^{\prime}(z), z^{2} q^{\prime \prime}(z), z^{3} q^{\prime \prime \prime}(z) ; z\right),
\end{aligned}
$$

which means that $q$ is the best dominant of (2.11).

\section{Special Cases}

We specialize the class of admissible functions and corresponding theorems for the case when $q(\mathbb{U})$ is the disk $\mathbb{U}_{M}:=\{w \in \mathbb{C}:|w|<M\}$. First, we remark that the function

$$
q(z)=M z, \quad M>0, z \in \mathbb{U}
$$

is univalent in $\overline{\mathbb{U}}$ and satisfies $q(\mathbb{U})=\mathbb{U}_{M}, q \in \mathcal{Q}_{0}$ and $E(q)=\emptyset$.

Definition 3.1 Let $\Omega$ be a subset of $\mathbb{C}$ and $q$ be given by (3.1). The class of admissible operators $\Phi_{p}[\Omega, M]$ consists of those functions $\phi: \mathbb{C}^{4} \times \mathbb{U} \rightarrow \mathbb{C}$ that satisfy the following admissibility condition:

$$
\phi\left(\alpha_{1}, \beta_{1}, L, N ; z\right) \notin \Omega
$$

whenever

$$
\begin{aligned}
& \alpha_{1}=\beta_{1}=M e^{i \theta}, \quad M>0 \\
& \operatorname{Re}\left(\frac{p}{\zeta^{2}} \frac{\left[L+(1-\zeta)\left(1-\zeta+\frac{\zeta}{p}\right) \alpha_{2}-\left(2-2 \zeta+\frac{\zeta}{p}\right) \beta_{2}\right]}{\alpha_{1}}+1\right) \geq p
\end{aligned}
$$

and

$$
\frac{p^{2}}{\zeta^{2}} \operatorname{Re}\left(\begin{array}{c}
N-3\left(1-\zeta+\frac{\zeta}{p}\right) L-(1-\zeta)\left(1-\zeta+\frac{\zeta}{p}\right)\left(1-\zeta+\frac{2 \zeta}{p}\right) \alpha_{2} \\
+\left[2\left(1-\zeta+\frac{\zeta}{p}\right)\left(2-2 \zeta+\frac{\zeta}{p}\right)-(1-\zeta)^{2}\right] \beta_{2}
\end{array}\right) \geq 0
$$

where $\operatorname{Re}\left(L e^{-i \theta}\right) \geq p(p-1) M$ and $\operatorname{Re}\left(N e^{-i \theta}\right) \geq 0$ for all $\theta \in[0,2 \pi]$ and $p \geq 2$. 
Using this definition of the class of admissible functions, from Theorem 2.1 we obtain the following result.

Corollary 3.1 Let $\Omega$ be a subset of $\mathbb{C}$ and $\phi \in \Phi_{p}[\Omega, M]$. If we suppose that

$$
\left|z\left(\mathcal{N}_{p, \lambda, \mu, \eta, \eta}^{k, \delta, \zeta} f(z)\right)^{\prime}\right| \leq p M, \quad z \in \mathbb{U}
$$

and the function $q$ is given by (3.1), then

$$
\phi\left(\mathcal{N}_{p, \lambda, \mu, \eta}^{k, \delta, \zeta} f(z), \mathcal{N}_{p, \lambda, \mu, \eta}^{k+1, \delta, \zeta} f(z), \mathcal{N}_{p, \lambda, \mu, \eta}^{k+2, \delta, \zeta} f(z), \mathcal{N}_{p, \lambda, \mu, \eta}^{k+3, \delta, \zeta} f(z) ; z\right) \in \Omega, \quad z \in \mathbb{U}
$$

implies

$$
\mathcal{N}_{p, \lambda, \mu, \eta}^{k, \delta, \zeta} f(z) \prec M z
$$

For the special case when $\Omega=q(\mathbb{U})=\{w \in \mathbb{C}:|w|<M\}$, Corollary 3.1 reduces to the following corollary.

Corollary 3.2 Let $\phi \in \Phi_{p}[q(\mathbb{U}), M]$ and suppose that the function q given by (3.1) satisfies condition (3.3). Then

$$
\left|\phi\left(\mathcal{N}_{p, \lambda, \mu, \eta}^{k, \delta, \zeta} f(z), \mathcal{N}_{p, \lambda, \mu, \eta}^{k+1, \delta, \zeta} f(z), \mathcal{N}_{p, \lambda, \mu, \eta}^{k+2, \delta, \zeta} f(z), \mathcal{N}_{p, \lambda, \mu, \eta}^{k+3, \delta, \zeta} f(z) ; z\right)\right|<M, \quad z \in \mathbb{U}
$$

implies

$$
\mathcal{N}_{p, \lambda, \mu, \eta}^{k, \delta, \zeta} f(z) \prec M z
$$

Let $\phi\left(\alpha_{1}, \beta_{1}, \gamma_{1}, \varepsilon_{1} ; z\right)=\alpha_{1}+\beta_{1}$ and $\Omega=h(\mathbb{U})$, where $h(z)=2 M z$. We will show that $\phi \in$ $\Phi_{p}[h(\mathbb{U}), M]$ by proving that condition (3.2) is satisfied. Thus,

$$
\left|\phi\left(\alpha_{1}, \beta_{1}, L, N ; z\right)\right|=\left|M e^{i \theta}+M e^{i \theta}\right|=2 M,
$$

where $\operatorname{Re}\left(L e^{-i \theta}\right) \geq p(p-1) M, \operatorname{Re}\left(N e^{-i \theta}\right) \geq 0$ for all $\theta \in[0,2 \pi]$ and $p \geq 2$.

Suppose that $A$ and $B$ are two complex-valued functions defined on $\mathbb{U}$ that satisfy $\operatorname{Re} A(z)>0, \operatorname{Re} B(z)>0$ for all $z \in \mathbb{U}$. Let $\phi\left(\alpha_{1}, \beta_{1}, \gamma_{1}, \varepsilon_{1} ; z\right)=1+A(z) \alpha_{1}+B(z) \beta_{1}$ and $\Omega=h(\mathbb{U})$, where $h(z)=z$. We will show that $\phi \in \Phi_{p}[h(\mathbb{U}), M]$ by proving that condition (3.2) is satisfied. Since

$$
\left|\phi\left(\alpha_{1}, \beta_{1}, L, N ; z\right)\right|=\left|1+A(z) M e^{i \theta}+B(z) M e^{i \theta}\right| \geq 1+M \operatorname{Re}[A(z)+B(z)]>1, \quad z \in \mathbb{U}
$$

where $\operatorname{Re}\left(L e^{-i \theta}\right) \geq p(p-1) M, \operatorname{Re}\left(N e^{-i \theta}\right) \geq 0$ for all $\theta \in[0,2 \pi]$ and $p \geq 2$.

Let $A: \mathbb{U} \rightarrow(1,+\infty), B: \mathbb{U} \rightarrow(0,+\infty), \phi\left(\alpha_{1}, \beta_{1}, \gamma_{1}, \varepsilon_{1} ; z\right)=\alpha_{1}+\beta_{1}+A(z) \gamma_{1}+B(z) \varepsilon_{1}$ and $\Omega=h(\mathbb{U})$, where $h(z)=4 M z$. We will show that $\phi \in \Phi_{p}[h(\mathbb{U}), M]$ by proving that condition (3.2) is satisfied. Thus,

$$
\left|\phi\left(\alpha_{1}, \beta_{1}, L, N ; z\right)\right|=\left|2 M e^{i \theta}+A(z) L+B(z) N\right|
$$




$$
\begin{aligned}
& =\left|2 M+A(z) L e^{-i \theta}+B(z) N e^{-i \theta}\right| \\
& \geq 2 M+A(z) \operatorname{Re}\left(L e^{-i \theta}\right)+B(z) \operatorname{Re}\left(N e^{-i \theta}\right) \\
& \geq 2 M+p(p-1) M A(z) \geq 2 M+p(p-1) M \geq 4 M, \quad z \in \mathbb{U},
\end{aligned}
$$

where $\operatorname{Re}\left(L e^{-i \theta}\right) \geq p(p-1) M, \operatorname{Re}\left(N e^{-i \theta}\right) \geq 0$ for all $\theta \in[0,2 \pi]$ and $p \geq 2$, from Corollary 3.2 we have the following special case.

Example 3.1 If $A: \mathbb{U} \rightarrow(1,+\infty), B: \mathbb{U} \rightarrow(0,+\infty)$ and $f \in \mathcal{A}(p)$ such that

$$
\left|z\left(\mathcal{N}_{p, \lambda, \mu, \eta}^{k, \delta, \zeta} f(z)\right)^{\prime}\right| \leq p M, \quad z \in \mathbb{U}
$$

then

$$
\left|\mathcal{N}_{p, \lambda, \mu, \eta}^{k, \delta, \zeta} f(z)+\mathcal{N}_{p, \lambda, \mu, \eta}^{k+1, \delta, \zeta} f(z)+A(z) \mathcal{N}_{p, \lambda, \mu, \eta}^{k+2, \delta, \zeta} f(z)+B(z) \mathcal{N}_{p, \lambda, \mu, \eta}^{k+3, \delta, \zeta} f(z)\right|<4 M, \quad z \in \mathbb{U}
$$

implies that

$$
\mathcal{N}_{p, \lambda, \mu, \eta}^{k, \delta, \zeta} f(z) \prec M z
$$

Let $A, B: \mathbb{U} \rightarrow \mathbb{C}$, with $\operatorname{Re}[A(z)+B(z)]>0$ for all $z \in \mathbb{U}$, let $C: \mathbb{U} \rightarrow(1,+\infty), D: \mathbb{U} \rightarrow$ $(0,+\infty), \phi\left(\alpha_{1}, \beta_{1}, \gamma_{1}, \varepsilon_{1} ; z\right)=A(z) \alpha_{1}+B(z) \beta_{1}+C(z) \gamma_{1}+D(z) \varepsilon_{1}$ and $\Omega=h(\mathbb{U})$, where $h(z)=$ $2 M z$. We will show that $\phi \in \Phi_{p}[h(\mathbb{U}), M]$ by proving that condition (3.2) is satisfied. Thus,

$$
\begin{aligned}
\left|\phi\left(\alpha_{1}, \beta_{1}, L, N ; z\right)\right| & =\left|M e^{i \theta} A(z)+M e^{i \theta} B(z)+C(z) L+D(z) N\right| \\
& =\left|M[A(z)+B(z)]+C(z) L e^{-i \theta}+D(z) N e^{-i \theta}\right| \\
& \geq M \operatorname{Re}[A(z)+B(z)]+C(z) \operatorname{Re}\left(L e^{-i \theta}\right)+D(z) \operatorname{Re}\left(N e^{-i \theta}\right) \\
& \geq p(p-1) M C(z) \geq p(p-1) M \geq 2 M, \quad z \in \mathbb{U},
\end{aligned}
$$

where $\operatorname{Re}\left(L e^{-i \theta}\right) \geq p(p-1) M, \operatorname{Re}\left(N e^{-i \theta}\right) \geq 0$ for all $\theta \in[0,2 \pi]$ and $p \geq 2$, from Corollary 3.2 we have the following special case.

Example 3.2 Let $A, B: \mathbb{U} \rightarrow \mathbb{C}$, with $\operatorname{Re}[A(z)+B(z)]>0$ for all $z \in \mathbb{U}$, and $C: \mathbb{U} \rightarrow(1,+\infty)$, $D: \mathbb{U} \rightarrow(0,+\infty)$. If $f \in \mathcal{A}(p)$ such that

$$
\left|z\left(\mathcal{N}_{p, \lambda, \mu, \eta}^{k, \delta, \zeta} f(z)\right)^{\prime}\right| \leq p M, \quad z \in \mathbb{U},
$$

then

$$
\begin{aligned}
& \left|A(z) \mathcal{N}_{p, \lambda, \mu, \eta}^{k, \delta, \zeta} f(z)+B(z) \mathcal{N}_{p, \lambda, \mu, \eta}^{k+1, \delta, \zeta} f(z)+C(z) \mathcal{N}_{p, \lambda, \mu, \eta}^{k+2, \delta, \zeta} f(z)+D(z) \mathcal{N}_{p, \lambda, \mu, \eta}^{k+3, \delta, \zeta} f(z)\right|<2 M, \\
& \quad z \in \mathbb{U}
\end{aligned}
$$

implies that

$$
\mathcal{N}_{p, \lambda, \mu, \eta}^{k, \delta, \zeta} f(z) \prec M z .
$$


Let $\phi\left(\alpha_{1}, \beta_{1}, \gamma_{1}, \varepsilon_{1} ; z\right)=1+\frac{\gamma_{1}}{\alpha_{1}}+\frac{\varepsilon_{1}}{\alpha_{1}}$ and $\Omega=\{w \in \mathbb{C}: \operatorname{Re} w<3\}$. We will show that $\phi \in$ $\Phi_{p}[\Omega, M]$ by proving that condition (3.2) is satisfied. Thus,

$$
\operatorname{Re} \phi\left(\alpha_{1}, \beta_{1}, L, N ; z\right)=\operatorname{Re}\left(1+\frac{L e^{-i \theta}}{M}+\frac{N e^{-i \theta}}{M}\right) \geq 1+p(p-1) \geq 3, \quad z \in \mathbb{U},
$$

where $\operatorname{Re}\left(L e^{-i \theta}\right) \geq p(p-1) M$ and $\operatorname{Re}\left(N e^{-i \theta}\right) \geq 0$ for all $\theta \in[0,2 \pi]$ and $p \geq 2$, from Corollary 3.2 we obtain the following.

Example 3.3 If $f \in \mathcal{A}(p)$ such that

$$
\left|z\left(\mathcal{N}_{p, \lambda, \mu, \eta, \eta}^{k, \delta, \zeta} f(z)\right)^{\prime}\right| \leq p M, \quad z \in \mathbb{U}
$$

then

$$
\operatorname{Re}\left(1+\frac{\mathcal{N}_{p, \lambda, \mu, \eta}^{k+2, \delta} f(z)}{\mathcal{N}_{p, \lambda, \mu, \eta}^{k, \delta, \zeta} f(z)}+\frac{\mathcal{N}_{p, \lambda, \mu, \eta}^{k+3, \eta, \zeta} f(z)}{\mathcal{N}_{p, \lambda, \mu, \eta, \eta}^{k, \delta,} f(z)}\right)<3, \quad z \in \mathbb{U},
$$

implies that

$$
\mathcal{N}_{p, \lambda, \mu, \eta}^{k, \delta, \zeta} f(z) \prec M z
$$

Remark 3.1 For different choices of $k, \delta, \zeta, p, \lambda, \mu$, and $\eta$, we will obtain new results for different operators defined in the introduction.

\section{Acknowledgements}

The authors are grateful to the reviewers of this article that gave valuable remarks, comments, and advice in order to revise and improve the results of the paper. Further, the first author would like to thank ITAM for the kind hospitality during her stay in Mexico.

\section{Funding}

Not applicable.

\section{Availability of data and materials}

Not applicable.

\section{Competing interests}

The authors declare that they have no competing interests.

\section{Authors' contributions}

Both authors contributed equally to this manuscript. All authors read and approved the final manuscript.

\section{Author details}

${ }^{1}$ Department of Mathematics, ITAM, Mexico City, Mexico. ${ }^{2}$ Department of Mathematics, Faculty of Science, Menofia University, Shebin Elkom, Egypt. ${ }^{3}$ Faculty of Mathematics and Computer Science, Babeş-Bolyai University, Cluj-Napoca, Romania.

\section{Publisher's Note}

Springer Nature remains neutral with regard to jurisdictional claims in published maps and institutional affiliations.

Received: 4 April 2019 Accepted: 2 September 2019 Published online: 11 September 2019

\section{References}

1. Al-Oboudi, F.M.: On univalent functions defined by a generalized Sălăgean operator. Int. J. Math. Math. Sci. 27, 1429-1436 (2004)

2. Antonino, J.A., Miller, S.S.: Third-order differential inequalities and subordinations in the complex plane. Complex Var. Theory Appl. 56(5), 439-454 (2011) 
3. Aouf, M.K.: Certain subclasses of multivalent prestarlike functions with negative coefficients. Demonstr. Math. 40(4), 799-814 (2007)

4. Aouf, M.K., Dziok, J.: Distortion and convolution theorem for operators of generalized fractional calculus involving Wright function. J. Appl. Anal. 14(2), 183-192 (2008)

5. Aouf, M.K., Mostafa, A.O.: On a subclass of $n-p$-valent prestarlike functions. Comput. Math. Appl. 55, 851-861 (2008)

6. Aouf, M.K., Mostafa, A.O., Zayed, H.M.: Subordination and superordination properties of $p$-valent functions defined by a generalized fractional differintegral operator. Quaest. Math. 39(4), 545-560 (2016)

7. Aouf, M.K., Mostafa, A.O., Zayed, H.M.: Some characterizations of integral operators associated with certain classes of $p$-valent functions defined by the Srivastava-Saigo-Owa fractional differintegral operator. Complex Anal. Oper. Theory 10, 1267-1275 (2016)

8. Aouf, M.K., Mostafa, A.O., Zayed, H.M.: On certain subclasses of multivalent functions defined by a generalized fractional differintegral operator. Afr. Math. 28, 99-107 (2017)

9. Bulboacă, T.: Differential Subordinations and Superordinations: New Results. House of Scientific Boook Publ., Cluj-Napoca (2005)

10. Goyal, G.P., Prajapat, J.K.: A new class of analytic $p$-valent functions with negative coefficients and fractional calculus operators. Tamsui Oxford Univ. J. Math. Sci. 20(2), 175-186 (2004)

11. Kamali, M., Orhan, H.: On a subclass of certain starlike functions with negative coefficients. Bull. Korean Math. Soc. 41(1), 53-71 (2004)

12. Miller, S.S., Mocanu, P.T.: Differential Subordinations: Theory and Applications. Series on Monographs and Textbooks in Pure and Appl. Math., vol. 255. Dekker, New York (2000)

13. Owa, S.: On the distortion theorems I. Kyungpook Math. J. 18, 53-59 (1978)

14. Owa, S., Srivastava, H.M.: Univalent and starlike generalized hypergeometric functions. Can. J. Math. 39, 1057-1077 (1987)

15. Prajapat, J.K., Aouf, M.K.: Majorization problem for certain class of $p$-valently analytic function defined by generalized fractional differintegral operator. Comput. Math. Appl. 63(1), 42-47 (2012)

16. Prajapat, J.K., Raina, R.K., Srivastava, H.M.: Some inclusion properties for certain subclasses of strongly starlike and strongly convex functions involving a family of fractional integral operators. Integral Transforms Spec. Funct. 18(9), 639-651 (2007)

17. Saigo, M.: A remark on integral operators involving the Gauss hypergeometric functions. Math. Rep. Coll. Gen. Educ. Kyushu Univ. 11, 135-143 (1978)

18. Sălăgean, G.S.: Subclasses of univalent functions. In: Complex Analysis—Fifth Romanian Finnish Seminar, Part 1, Bucharest, 1981. Lecture Notes in Math., pp. 362-372. Springer, Berlin (1013)

19. Srivastava, H.M., Aouf, M.K., Mostafa, A.O., Zayed, H.M.: Certain subordination-preserving family of integral operators associated with p-valent functions. Appl. Math. Inf. Sci. 11(4), 951-960 (2017)

20. Srivastava, H.M., Owa, S.: Some characterizations and distortions theorems involving fractional calculus, generalized hypergeometric functions, Hadamard products, linear operators and certain subclasses of analytic functions. Nagoya Math. J. 106, 1-28 (1987)

21. Srivastava, H.M., Prajapati, A., Gochhayat, P.: Third-order differential subordination and differential superordination results for analytic functions involving the Srivastava-Attiya operator. Appl. Math. Inf. Sci. 12(3), 469-481 (2018)

22. Srivastava, H.M., Saigo, M., Owa, S.: A class of distortion theorems involving certain operators of fractional calculus, J. Math. Anal. Appl. 131, 412-420 (1988)

23. Srivastava, H.M., Sharma, P., Raina, R.K.: Inclusion results for certain classes of analytic functions associated with a new fractional differintegral operator. Rev. R. Acad. Cienc. Exactas Fís. Nat., Ser. A Mat. 112, 271-292 (2018)

24. Tang, H., Deng, G.-T., Li, S.-H., Aouf, M.K.: Inclusion results for certain subclasses of spiral-like multivalent functions involving a generalized fractional differintegral operator. Integral Transforms Spec. Funct. 24(11), 873-883 (2013)

25. Tang, H., Deniz, E.: Third-order differential subordination results for analytic functions involving the generalized Bessel functions. Acta Math. Sci. Ser. B Engl. Ed. 34, 1707-1719 (2014)

26. Tang, H., Srivastava, H.M., Deniz, E., Li, S.: Third-order differential superordination involving the generalized Bessel functions. Bull. Malays. Math. Sci. Soc. 38, 1669-1688 (2015)

\section{Submit your manuscript to a SpringerOpen ${ }^{\circ}$ journal and benefit from:}

- Convenient online submission

- Rigorous peer review

- Open access: articles freely available online

- High visibility within the field

- Retaining the copyright to your article

Submit your next manuscript at $\gg$ springeropen.com 